\title{
Transplantation for ultra high-risk neuroblastoma patients: effect of tandem autologous stem cell transplantation
}

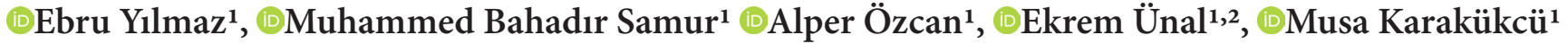 \\ ${ }^{1}$ Erciyes University, Faculty of Medicine, Department of Pediatrics, Division of Pediatric Hematology and Oncology \& Pediatric HSCT Unit, \\ Kayseri, Turkey \\ ${ }^{2}$ Erciyes University Betül Ziya EREN Genome and Stem Cell, Department of Molecular Biology and Genetics, Kayseri, Turkey
}

Cite this article as: Yılmaz E, Samur MB, Özcan A, Ünal E, Karakükcü M. Transplantation for ultra high-risk neuroblastoma patients: effect of tandem autologous stem cell transplantation. J Health Sci Med 2021; 4(6): 943-948.

\begin{abstract}
Aim: Neuroblastoma is a tumor with a high mortality rate originating from primitive sympathetic ganglion cells. In recent years, the definition of "ultra high-risk" has been used, in which the response to treatment is added in addition to the disease characteristics. In this study, the results of tandem high-dose Iodine $\left.131{ }^{131} \mathrm{I}\right)$ metaiodobenzylguanidine (MIBG) and tandem autologous hematopoietic stem cell transplantation (HSCT) in patients who were diagnosed as refractory after first-line treatments and considered with ultra-high risk nöroblastom (NBL) in Erciyes University Medical Faculty Pediatric Bone Marrow Transplant Center were shared.

Material and Method: Eleven patients who underwent tandem MIBG and autologous HSCT with the diagnosis of refractory/ ultra high-risk NBL between 2011 and 2021 were included in the study. Patient's data were obtained retrospectively using an electronic or manuscript medical record.

Results: Tandem autologous HSCT and MIBG treatment were applied to 11 patients with a median age of 40.5 months who were diagnosed with refractory/ultra high-risk neuroblastoma. In our study, the overall survival rate was $54.5 \%$ and eventfree survival (EFS) was 39\% at a median follow-up of 20.7 (7.8-105.6) months. Of the 6 surviving patients, 4 are followed in complete remission and 2 in partial remission.

Conclusion: Our study is the only study that includes a group of patients who received tandem high-dose MIBG and tandem autologous HSCT transplantation in refractory NBL patients without cytokine and immunotherapy. Tandem transplantation and MIBG therapy in ultra-high-risk NBL cases remain promising with acceptable toxicity. Adding immunotherapy to this treatment protocol and combining it with new treatment modalities such as anaplastic lymphoma kinase (ALK), which also takes into account genetic characteristics, may increase survival and event-free survival rates.
\end{abstract}

Keywords: Neuroblastoma, tandem stem cell transplantation, children, tandem MIBG

\section{INTRODUCTION}

The spectrum of neuroblastic tumors (this term includes neuroblastomas, ganglioneuroblastomas, and ganglioneuromas) is one of the most common lethal malign diseases that arise from primitive sympathetic ganglion cells (1). Neuroblastoma (NBL) is the most common extracranial malignancy overall in the first year of life as the cause of $15 \%$ mortality of childhood cancers and disease is characterized by a heterogeneous group of histopathologic appearance, biologic characteristics with onset usually before age 5 years old (2-4). Neuroblastomas constitute $13 \%$ of childhood cancers in Turkey (5). Remarkably divergent courses of tumors are typically associated with patient age, tumor stage, and histology, genetic and chromosomal abnormalities (6). Global surveys estimate the occurrence of NBL in the range of eight to ten per million children $0-14$ years $(2,3)$.

NBL remains distinct from other solid tumors. The disease has ranged from extremely aggressive metastatic disease to spontaneous regression forms, this is probably due to their unique histological and biological character (6). While the most adrenal gland involvement was observed ( $40 \%$ ), up to $25 \%$ followed by abdominal, $15 \%$ thoracic, $10 \%$ other nervous sympathetic systems; location of the tumor determines the symptoms including abdominal mass or pain, weakness, constipation and bladder dysfunction, palpable non-tender subcutaneous nodules, anemia, and bone pain. Elevated levels of catecholamines 
may lead to significant morbidity and death but also "atypical" presentation such as horner syndrome, proptosis, periorbital ecchymoses, or opsoclonus myoclonus syndrome expected $(1,2,6)$. Previous studies have shown that the most robust prognostic factors are age, stage, metastasis, histology, Stage of the tumor at the time of diagnosis, and amplification of the N-Myc oncogene (7). Markers associated with a poor prognosis include ferritin, lactate dehydrogenase ( $\mathrm{LDH})$, and neuron-specific enolase (NSE). However, these markers have become less important due to the discovery of $\mathrm{N}$-Myc amplification, deletion of chromosome 1, and heterozygosity at $11 \mathrm{q} 23(1,7)$.

Standard care of high-risk NBL is defined as multi-modal treatment which includes induction chemotherapy, surgical tumor resection, immunotherapy, consolidative highdose chemotherapy with autologous hematopoietic stem cell transplantation (HSCT), post-consolidation therapy to treat minimal residual disease including isotretinoin, post-transplant radiotherapy $(8,9,10)$. Detection of GD2 and ALK expression in neuroblastoma cells brings up alternative treatment options (11). Survival is around less than $\% 15$ before these intense therapies but, it is still below $50 \%$ despite all these treatment regimens $(9,10)$. The use of high-dose chemotherapy with autologous HSCT rescue and Iodine 131 (131) metaiodobenzylguanidine (MIBG) has still insufficient in survival however, patients with the high-risk disease requires treatment with multi-modal therapy (3). Especially, refractory and ultra high-risk patients (which including treatment response) have an extremely poor prognosis; despite gradual improvements chemotherapy, surgery, radiotherapy, and HSCT with $\% 10-15$ survival $(8,12)$.

Studies over the past few years show that survival of $\mathrm{NBL}$ patients undergoing tandem transplantation is significantly better, by the time tandem HSCTs are getting the standard first-line therapy in The United States of America step by step $(8,13,14)$. There are still institutes that apply myeloablative regimens consolidated with immunotherapy and isotretinoin as standard therapy (15). Thus, improvement of treatment strategies of stem cells is warranted.

In this study, the results of tandem high-dose MIBG and tandem autologous HSCT in patients who were diagnosed as refractory after first-line treatments and considered with ultra-high risk NBL in Erciyes University Medical Faculty Pediatric Bone Marrow Transplant Center were shared. In comparison to previously published bone marrow transplantation experience, our data is the only study with both tandem high-dose MIBG and tandem autologous HSCT transplantation for pediatric ultra high-risk neuroblastoma patients.

\section{MATERIAL AND METHOD}

\section{Patients}

Eleven patients who underwent tandem MIBG and autologous HSCT with the diagnosis of refractory/ultra high-risk neuroblastoma at Erciyes University Pediatrics Bone Marrow Transplant Center between January 2011 and January 2021 were included in the study. Patient's data were obtained retrospectively using an electronic or manuscript medical record.

The study was carried out with the permission of Erciyes University Faculty of Medicine Clinical Researchs Ethics Committee (Date: 08.07.2020, Decision No: 2020/371). All procedures were carried out in accordance with the ethical rules and the principles of the Declaration of Helsinki.

\section{Diagnosis, Risk Classification, Genetic Study}

All patients were diagnosed with tissue biopsy. Molecular analyzes were performed on tissue samples taken at the time of diagnosis at Dokuz Eylül University Oncology Institute. Bone marrow aspiration and biopsy were used for staging the disease, MIBG, positron emission tomography/computed tomography (PET CT) and/or whole-body magnetic resonance imaging (MRI) were used as imaging modalities. In the evaluation of patients' response to treatment, biochemical parameters such as lactate dehydrogenase, ferritin, NSE; if there is bone marrow involvement at the time of diagnosis or if additional symptoms developed, bone marrow aspiration and biopsy; MIBG, PET CT and/or MRI were used as imaging modalities. The patients were staged according to the International Neuroblastoma Staging System (INSS) (16). The patients' response to treatment was evaluated using the international neuroblastoma response criteria (17).

\section{Treatment Protocols}

All patients received the Turkish Pediatrics Oncology Group Neuroblastoma 2009 (TPOG 2009) treatment protocol. Tumor resection was performed before and/ or after the protocol according to the size, localization and invasion of the tumor. Interim evaluation was made after the $3^{\text {rd }}$ or $4^{\text {th }}$ cycle. Following the interim evaluation, hematopoietic stem cells were collected from the patient with the help of an apheresis device using a central venous catheter. During the stem cell collection stage, granulocyte colony-stimulating factor (GCSF) was administered at a dose of $10 \mathrm{mcg} / \mathrm{kg}$ to the patients. Enough cells for two transplants were collected and cryopreserved. $8-12 \mathrm{mCi} / \mathrm{kg}$ dose of MIBG was given to the patients who completed the induction treatment and underwent surgery.The first transplantation was performed with conditioning regimen consisting of busulfan and melphalan 3 weeks after the first MIBG. 
MIBG was repeated at a dose of $8-12 \mathrm{mCi} / \mathrm{kg}$ after first autologous HSCT in patients. In the second autologous HSCT, a conditioning regimen containing melphalan, etoposide and carboplatin was used. Nine cycles of 13-cis retinoic acid were given in the post-transplant maintenance treatment (Figure 1).

\section{Statistical Analysis}

Categorical data were expressed as the number of patients and percentage and continuous variables were expressed as the mean \pm standard deviation or median (minimummaximum). Nominal variables were expressed as number of cases and percentage (\%). The Kaplan-Meier method was used to estimate survival probabilities.

\section{RESULTS}

The median age of 11 patients ( 8 boys, 3 girls) included in the study was 40.5 (range, 22.1-72.9) months. The clinical features of the patients are summarized in Table 1.

The median value of CD34 (+) cell count in the stem cell product given in the first transplantation was 7.5 (range, $4.55-17.1) \times 10^{6}$ cells $/ \mathrm{kg}$. The median day of myeloid and platelet engraftment was detected as +12 (9-22) days and +18 (12-28) days, respectively. The mean time between two HSCT was $4.7 \pm 0.5$ months. In the second HSCT, 5.7 (2.7-16.7) $\times 10^{6}$ cells/kg CD34(+) cells were given. The median day of myeloid and platelet engraftment was +11 (9-15) days and +19 (16-21) days, respectively.

Only one patient did not have bone metastases at the time of diagnosis (Patient 6). One patient died on the $45^{\text {th }}$ day of the second transplantation due to venoocclusive disease and sepsis, and 4 patients died due to progressive disease. Transplant-related mortality was $4.5 \%$. After the second HSCT, 3 patients (Patient 9, 10, and 11) with residual tumors were given radiotherapy for local control. Retinoic acid maintenance therapy was given to the surviving patients (Patient 2, 5, 8, 9, 10, and 11).

In our study, the overall survival rate (OS) was $54.5 \%$ and event-free survival (EFS) was $39 \%$ at a median followup of 20.7 (range, 7.8-105.6) months (Figure 2). Of the 6 surviving patients, 4 are followed in complete remission and 2 in partial remission (Table $\mathbf{1}$ ).

\begin{tabular}{|ccccccccc|}
\hline $\begin{array}{c}\text { Table 1. Patient characteristics } \\
\text { Patient } \\
\text { number }\end{array}$ & Sex & $\begin{array}{c}\text { Diagnosis age } \\
\text { (month) }\end{array}$ & Tumor type & Histology & Stage & N-Myc & Outcome \\
\hline 1 & Boy & 26.7 & Ganglioneuroblastoma & Poorly Differentiated & 4 & Amplified & Exitus \\
2 & Boy & 24.4 & Neuroblastoma & Intermixed & 4 & Amplified & Remission \\
3 & Boy & 24 & Neuroblastoma & Undiferantiated & 4 & Not amplified & Exitus \\
4 & Girl & 35.9 & Neuroblastoma & Undiferantiated & 4 & Not amplified & Exitus \\
5 & Boy & 45.6 & Neuroblastoma & Undiferantiated & 4 & Not amplified & Remission \\
6 & Boy & 13.7 & Neuroblastoma & Undiferantiated & 4 & Amplified & Exitus \\
7 & Girl & 88.2 & Neuroblastoma & Undiferantiated & 4 & Not amplified & Exitus \\
8 & Girl & 31.7 & Ganglioneuroblastoma & Poorly Differentiated & 4 & Not amplified & Remission \\
9 & Boy & 13.1 & Neuroblastoma & Undiferantiated & 4 & Amplified & Remission \\
10 & Boy & 33.8 & Neuroblastoma & Poorly Differentiated & 4 & Amplified & Partial Remission \\
11 & Boy & 48.8 & Ganglioneuroblastoma & Poorly Differentiated & 4 & Not amplified & Partial Remission \\
\hline
\end{tabular}

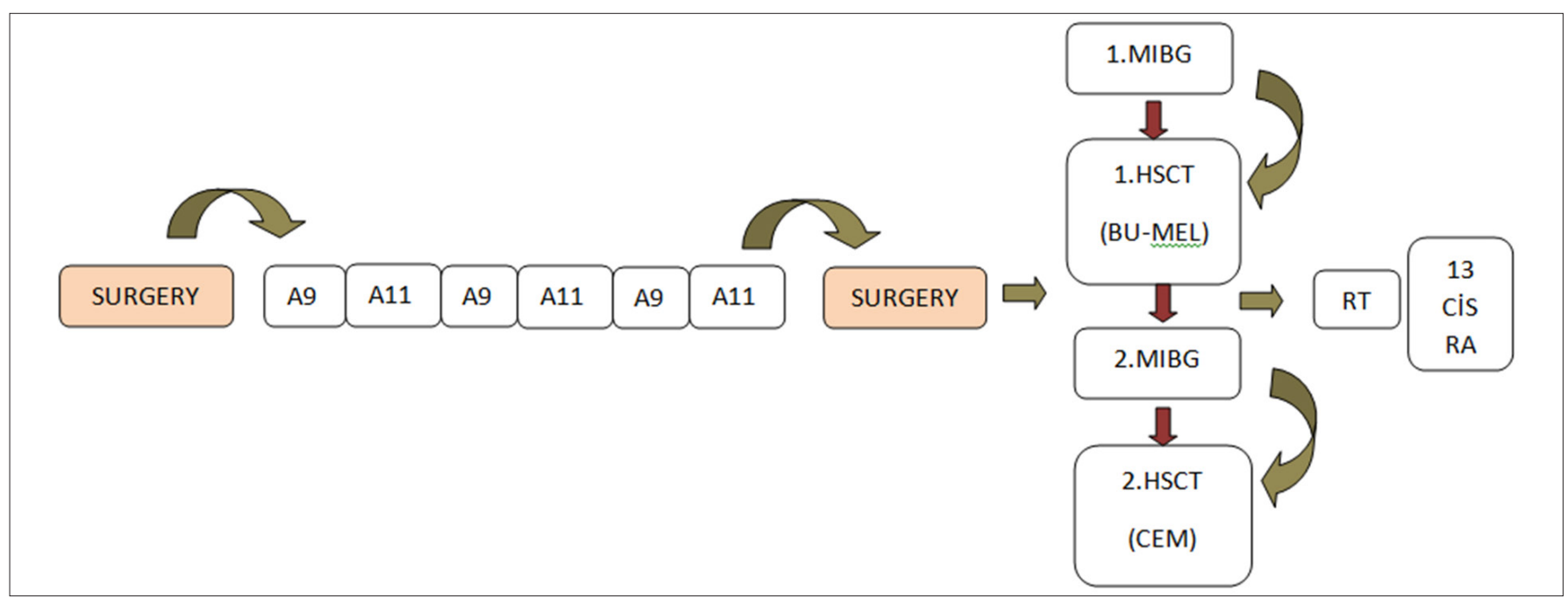

Figure 1. Treatment scheme

A9: Vincristine, dacarbazine, ifosfamide, adriamycin; A11: Cyclophosphamide, etoposide, cisplatin; Bu: Busulfan; Mel: Melphalan; CEM: Carboplatin, etoposide, melphalan; HSCT: Hematopoietic stem cell transplantation; MIBG: Iodine 131 (131I) metaiodobenzylguanidine ; RT: Radiotherapy; 13 CÍS RA: 13-cis retinoic acid 


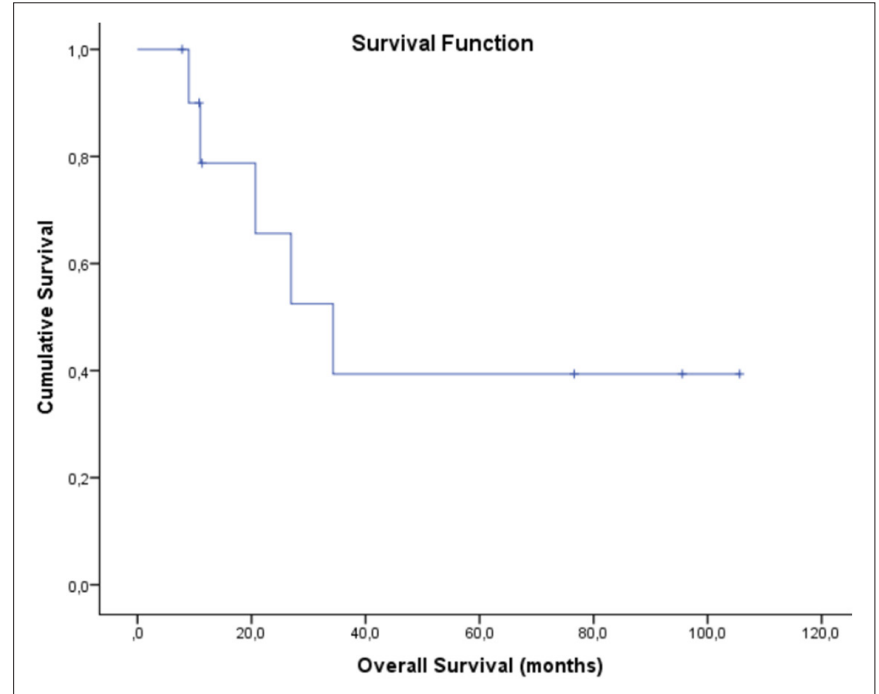

Figure 2. Overall survival of patients

\section{DISCUSSION}

Neuroblastoma is related to the disproportionate morbidity and mortality among the cancers of childhood and has been shown that high-dose chemotherapy with autologous stem cell rescue has improved the outcomes for children with high-risk neuroblastoma $(8,18)$. In non-metastatic patients, chemotherapy, surgery, and/ or radiotherapy are often cured. However, $40 \%$ of these patients are high-risk or ultra high-risk cases which is extremely difficult to provide cures for these patients. The current therapies including high-dose chemotherapy and HSCT are border on tolerability limits with insufficient effectiveness. Therefore, alternative protocols aiming at curative treatment are required.

The high-risk tumors include a heterogeneous group of patients with a wide range of outcome spectrum. Treatment of 5 phases with high-risk tumors includes; induction, surgery, metastatic remission, myeloablative chemotherapy with autologous HSCT, radiotherapy, and biological differentiation therapy (19). Main modalities have been standardized however, autologous HSCT approaches vary considerably. In the literature, autologous HSCT was performed with MIBG to be applied once to patients in the high-risk group, and the overall response rate was found to be $27 \%$ with the $31 \%$ 3-year EFS (20). In 2012, Qayed et al., showed that patients who were randomized to high dose chemotherapy and HSCT had a significantly improved at 3-year EFS compared with high dose chemotherapy and HSCT (4-year EFS 59.3 $\pm 6.7 \%$ vs. $26.8 \pm 9.2 \%, \mathrm{p}=0.01)$ (21). The Children's Oncology Group (COG) Phase 3 trial (NCT00567567) compared carboplatin-etoposide-melphalan and thiotepa based tandem HSCT. They found significantly increased 3 year EFS (3-year) scores (61.8\% versus 48.8\%; $\mathrm{p}=.0082)(14)$. Follow up results of this randomized controlled trial data in 2019 comparing tandem and single transplants show 85 of 176 tandem HSCT were in complete remission/ very good partial response, and 121 of 176 were needed immunotherapy (8). Another immunologically based study compared post-transplant dinutuximab beta effects was also concluded that improved EFS (5-year) results with superior significant improvement in OS (5-year) (66.7\% versus $11.4 \% ; \mathrm{p}=.0007)$ (22). Major tandem studies are summurized in Table 2 (8,23-27). The experience of allogeneic HSCT in high-risk neuroblastoma patients is increasing over time $(27,28)$.

In our study, the overall survival rate of ultra high-risk patients was $54.5 \%$ and EFS was $39 \%$ without cytokine and immunotherapies. In our patients with minimal toxicity, this tandem treatment modality provides encouraging results. Our results appear to be better than the previously high-risk studies eventhough immunotherapies were not used at all. MIBG is an adrenal imaging analogue marker initially developed at the University of Michigan fifty years ago; however, by the time with studies as therapy has directed on palliation for patients with refractory disease. The majority of authors focusing on patients with MIBG avid, non-responsive or progressive disease with safety profile, and also potential efficacy

Table 2. Tandem Hematopoietic Stem Cell Transplantation Data

\begin{tabular}{|c|c|c|c|c|c|c|}
\hline Study & Patient & $\begin{array}{l}\text { Pre-transplant } \\
\text { remission status }\end{array}$ & Conditioning regimen & $\begin{array}{c}\text { Additional } \\
\text { treatment }\end{array}$ & OS & EFS \\
\hline Park et al. & 176 & $\mathrm{PR}, \mathrm{CR}, \mathrm{MR}$ & СУ-TT/CEM & RT, 13 Cis RA, IT & 74\% (3-year) & $61 \%$ (3-year) \\
\hline Pasqualini et al. & 26 & PR, CR & TT/ Bu-Mel & $\mathrm{RT}, 13$ Cis RA & $69 \%$ (3-year) & $37.3 \%$ (3-year) \\
\hline Suh et al. & 18 & $\mathrm{PR}, \mathrm{CR}, \mathrm{MR}$ & CEM/Bu-Mel/TT-Cy & $\begin{array}{c}\text { RT, } 13 \text { Cis RA, single } \\
\text { MIBG, IT }\end{array}$ & $83 \%$ (5-year) & $64 \%$ (5-year) \\
\hline Lee et al. & 54 & PR, CR, MR & CEC/TT-Mel & $\begin{array}{c}\text { RT, } 13 \text { Cis RA, single } \\
\text { MIBG, IT }\end{array}$ & $72.4 \%$ (5-year) & $58.3 \%$ (5-year) \\
\hline Sung et al. & 50 & PR, CR, MR & $\begin{array}{c}\text { CEC/TT-Mel-TBI/ } \\
\text { TT-Mel }\end{array}$ & RT, 13 Cis RA, IT & 77\% (5-year) & $71.4 \%$ (5-year) \\
\hline George et al. & 82 & - & CEC/TBI-Mel & RT, 13 Cis RA & $64 \%$ (5-year) & $54 \%$ (5-year)-PFS \\
\hline Our Data & 11 & $\mathrm{PR}, \mathrm{PD}$ & $\mathrm{Bu}-\mathrm{Mel} / \mathrm{CEM}$ & $\begin{array}{l}\text { RT, } 13 \text { Cis RA, } \\
\text { tandem MIBG }\end{array}$ & $54.5 \%$ (3-year) & $39 \%$ (3-year) \\
\hline
\end{tabular}


of this regimen, the combination of chemotherapy and stem cell rescue is being considered within COG for the refractory neuroblastoma patients (29). As a result, our study suggested that tandem MIBG and tandem autologous HSCT combination might indeed improve the EFS of the ultra high-risk refractory patients.

The limitations of the study were that it is retrospective design and the sample is relatively small. In addition, the patients were not given cytokine and immunotherapy/ anti-GD2 antibodies. Survivors should be evaluated for any toxicity.

\section{CONCLUSION}

This study is the only case series study with both tandem high-dose MIBG and tandem autologous HSCT transplantation in refractory NBL patients without cytokine and immunotherapy. Tandem transplantation and MIBG therapy in ultra high-risk NBL cases remain promising with acceptable toxicity. Our data show that a significant contribution may be added to the survival of ultra high-risk NBL patients based on this dual protocol. Adding immunotherapy to this treatment protocol and combining it with new treatment modalities such as anaplastic lymphoma kinase (ALK), which also takes into account genetic characteristics, may increase survival and EFS rates.

\section{ETHICAL DECLARATIONS}

Ethics Committee Approval: The study was carried out with the permission of Erciyes University Faculty of Medicine Clinical Researchs Ethics Committee (Date: 08.07.2020, Decision No: 2020/371).

Informed Consent: Because the study was designed retrospectively, no written informed consent form was obtained from patients.

Referee Evaluation Process: Externally peer-reviewed.

Conflict of Interest Statement: The authors have no conflicts of interest to declare.

Financial Disclosure: The authors declared that this study has received no financial support.

Author Contributions: All of the authors declare that they have all participated in the design, execution, and analysis of the paper, and that they have approved the final version.

Acknowledgment: The authors thank Prof. Dr Mehmet Akif Özdemir, Prof. Dr. Türkan Patıroğlu for their valuable contributions to the study.

\section{REFERENCES}

1. Swift CC, Eklund MJ, Kraveka JM, Alazraki AL. Updates in Diagnosis, Management, and Treatment of Neuroblastoma. Radiographics 2018; 38: 566-80.
2. Schwab M, Shimada H, Joshi V, Brodeur GM. Neuroblastic tumours of adrenal gland and sympathetic nervous system. In: Pathology and Genetics. Tumours of the Nervous system, Kleihues P, Cavenee WK (eds). IARC Press: Lyon; 2000: 153-61.

3. Smith V, Foster J. High-risk neuroblastoma treatment review. Children (Basel) 2018; 5: 114.

4. Almandoz JP, Xie L, Schellinger JN, et al. Impact of COVID-19 stay-at-home orders on weight-related behaviours among patients with obesity. Clin Obes 2020; 10: e12386.

5. Kebudi R. Turkish Pediatric Oncology Group. Pediatric oncology in Turkey. J Pediatr Hematol Oncol 2012; 34: S12-4.

6. Louis CU, Shohet JM. Neuroblastoma: molecular pathogenesis and therapy. Annu Rev Med 2015; 66: 49-63.

7. Zhang Y, Huang D, Zhang W, et al. Clinical characteristics of infant neuroblastoma and a summary of treatment outcome. Oncol Lett 2016; 12: 5356-62.

8. Park JR, Kreissman SG, London WB, et al. Effect of tandem autologous stem cell transplant vs single transplant on event-free survival in patients with high-risk neuroblastoma: a randomized clinical trial. JAMA 2019; 322: 746-55.

9. Pinto NR, Applebaum MA, Volchenboum SL, et al. Advances in risk classification and treatment strategies for neuroblastoma. J Clin Oncol 2015; 33: 3008-17.

10.Smith V, Foster J. High-risk neuroblastoma treatment review. Children (Basel) 2018; 5.

11. Aygün Z, Batur Ş, Emre Ş, Celkan T, Özman O, Comunoglu N. Frequency of ALK and GD2 expression in neuroblastoma. Fetal Pediatr Pathol 2019; 38: 326-34.

12. Morgenstern DA, Bagatell R, Cohn SL, et al. The challenge of defining "ultra-high-risk" neuroblastoma. Pediatr Blood Cancer 2019; 66: e27556.

13. Berthold F, Spix C, Kaatsch P, Lampert F. Incidence, survival, and treatment of localized and metastatic neuroblastoma in Germany 1979-2015. Paediatr Drugs 2017; 19: 577-93.

14. Park JR, Kreissman SG, London WB, et al. A phase III randomized clinical trial (RCT) of tandem myeloablative autologous stem cell transplant (ASCT) using peripheral blood stem cell (PBSC) as consolidation therapy for high-risk neuroblastoma (HR-NB): A Children's Oncology Group (COG) study. J Clin Oncol 2016; 34.

15. Kushner BH, Ostrovnaya I, Cheung IY, et al. Lack of survival advantage with autologous stem-cell transplantation in high-risk neuroblastoma consolidated by anti-GD2 immunotherapy and isotretinoin. Oncotarget 2016; 7: 4155.

16. Kushner BH, Cheung NK, LaQuaglia MP, et al. International neuroblastoma staging system stage 1 neuroblastoma: a prospective study and literature review. J Clin Oncol 1996; 14: 2174-80.

17. Brodeur GM, Pritchard J, Berthold F, et al. Revisions of the international criteria for neuroblastoma diagnosis, staging, and response to treatment. J Clin Oncol 1993; 11: 1466-77.

18. Elborai Y, Hafez H, Moussa EA, et al. Comparison of toxicity following different conditioning regimens (busulfan/melphalan and carboplatin/etoposide/melphalan) for advanced stage neuroblastoma: Experience of two transplant centers. Pediatr Transplant 2016; 20(2): 284-9.

19. Bansal D, Totadri S, Chinnaswamy G, et al. Management of neuroblastoma: ICMR consensus document. Indian J Pediatr 2017; 84: 446-55.

20. Matthay KK, Tan JC, Villablanca JG, et al. Phase I dose escalation of iodine-131-metaiodobenzylguanidine with myeloablative chemotherapy and autologous stem-cell transplantation in refractory neuroblastoma: a new approaches to Neuroblastoma Therapy Consortium Study. J Clin Oncol 2006; 24: 500-6.

21. Qayed M, Chiang KY, Ricketts R, et al. Tandem stem cell rescue as consolidation therapy for high-risk neuroblastoma. Pediatr Blood Cancer 2012; 58: 448-52. 
22. Wawrzyniak-Dzierżek E, Gajek K, Rybka B, et al. Feasibility and safety of treosulfan, melphalan, and thiotepa-based megachemotherapy with autologous or allogeneic stem cell transplantation in heavily pretreated children with relapsed or refractory neuroblastoma. Biol Blood Marrow Transplant 2019; 25: 1792-7.

23. Pasqualini C, Dufour C, Goma G, Raquin MA, Lapierre V, ValteauCouanet D. Tandem high-dose chemotherapy with thiotepa and busulfan-melphalan and autologous stem cell transplantation in very high-risk neuroblastoma patients. Bone Marrow Transplant 2016; 51: 227-31.

24. Suh JK, Koh KN, Min SY, et al. Feasibility and effectiveness of treatment strategy of tandem high-dose chemotherapy and autologous stem cell transplantation in combination with 131 I-MIBG therapy for high-risk neuroblastoma. Pediatr Transplant 2020; 24: e13658.

25.Lee JW, Lee S, Cho HW, et al. Incorporation of highdose ${ }^{131}$ I-metaiodobenzylguanidine treatment into tandem highdose chemotherapy and autologous stem cell transplantation for high-risk neuroblastoma: results of the SMC NB-2009 study. J Hematol Oncol 2017; 10: 108.

26.Sung KW, Son $\mathrm{MH}$, Lee $\mathrm{SH}$, et al. Tandem high-dose chemotherapy and autologous stem cell transplantation in patients with high-risk neuroblastoma: results of SMC NB-2004 study. Bone Marrow Transplant 2013; 48: 68-73.

27.George RE, Li S, Medeiros-Nancarrow C, et al. High-risk neuroblastoma treated with tandem autologous peripheral-blood stem cell-supported transplantation: long-term survival update. J Clin Oncol 2006; 24: 2891-6.

28.Erbey F, Atay D, Akçay A, Öztürk G. Autologous cord blood transplantation in neuroblastoma. Turk J Pediatr 2015; 57: 210-1.

29. Grupp SA, Asgharzadeh S, Yanik GA. Neuroblastoma: issues in transplantation. Biol Blood Marrow Transplant 2012; 18: S92-100. 